\title{
Mutation model for nucleotide sequences based on crystal basis
}

\author{
C. Minichini, A. Sciarrino ${ }^{\dagger}$ \\ Dipartimento di Scienze Fisiche, Università di Napoli "Federico II" \\ and ${ }^{\dagger}$ I.N.F.N., Sezione di Napoli \\ Complesso Universitario di Monte S. Angelo \\ Via Cintia, I-80126 Naples, Italy
}

\begin{abstract}
A nucleotides sequence is identified, in the two (four) letters alphabet, by the the labels of a vector state of an irreducible representation of $\mathcal{U}_{q \rightarrow 0}(\operatorname{sl}(2))\left(\mathcal{U}_{q \rightarrow 0}(\operatorname{sl}(2) \oplus \operatorname{sl}(2))\right)$. A master equation for the distribuion function is written, where the intensity of the one-spin flip is assumed to depend from the variation of the labels of the state. In the two letters approximation, the numerically computed equilibrium distribution for short sequences is nicely fitted by a Yule distribution, which is the observed distribution of the ranked short oligonucleotides frequency in DNA. The four letter alphabet description, applied to the codons, is able to reproduce the form of the fitted rank ordered usage frequencies distribution.
\end{abstract}

Keywords: mutation, rank ordered distribution, codons, oligonucleotides, crystal basis

preprint DSF 14/2005

email: minichini@na.infn.it sciarrino@na.infn.it

Corresponding author: A. Sciarrino - Tel +39-081676807 - Fax +39-081676346 



\section{Introduction}

Mutations in DNA play a very important role in the theory of evolution. DNA and RNA are build up as sequences of four basis or nucleotides which are usually identified by the letters: C, G, T, A ( $\mathrm{T}$ being replaced by $\mathrm{U}$ in RNA), $\mathrm{C}$ and $\mathrm{U}$ ( $\mathrm{G}$ and $\mathrm{A}$ ) belonging to the purine family, denoted by $\mathrm{R}$ (respectively to the pyrimidine family, denoted by $\mathrm{Y}$ ). Therefore in the case of genome sequences each point in the sequence should be identified by an element of a four letter alphabet or by a set of two binary values. In a simplified treatment one identifies each element according to the purine or pyrimidine nature, reducing so to a two letter alphabet or to a binary set. Genetic mutations, i.e. modifications of the DNA genomic sequences, play a fundamental role in the evolution. They include changes of one or more than one nucleotide, insertions and deletions of nucleotides, frameshifts and inversions. In the present paper we consider only the point mutations, for a review see (Li Wen-Hsiung, 1997). These are usually modeled by stationary, homogeneous Markov process, which assume: 1) the nucleotide positions are stochastically independent one from another, which is clearly not true in functional sequences; 2) the mutation is not depending on the site and constant in time, which ignores the existence of "hot spots" for mutations as well as the probable existence of evolutionary spurts ; 3) the nucleotide frequencies are equilibrium frequencies. In the simplest model one can think of, all the mutations are assumed reversible and with equal rate, therefore only one parameter rules all the transitions. This is clearly a very rough approximation and indeed more complicated models have been proposed depending on more parameters. The most general one, with not reversible transitions, depending on the type of the nucleotide undergoing a mutation and on the kind of mutation, requires 12 parameters. However all these models are based on the assumption that the transitions are not depending of the neighbour nucleotides. In the early nineties was realized that the intensity of point mutations is really depending on the context where they happen (Blake R., Hess and Nicholson-Tuell, 1992; Hess, Blake J. and Blake R., 1994) and in the last decades an increasing amount of data in genetic research has provided further evidence that there is indeed a not negligible effect of the nearest neighbors as well as an effect of the the whole sequence, see e.g. (Arndt, Burge and Hwa, 2002). In the more simplified descriptions, where the elements of the two chemical families (purine and pyrimidine), the four nucleotides belong to, are identified, a correspondence is made between the nucleotides and the elements of a binary set. It follows that the mutations are mathematically modelised as transitions between binary labels sequences. As a binary alphabet is equivalent to spin variables, it is clear that the spin approaches, extensively studied in physics, have a natural application in the theory of molecular biological evolution. Indeed since 1986, when Leuthäusser (Leuthäusser, 1986, 1987) put a correspondence between the Eigen model of evolution (Eigen 1971; Eigen, McCaskill and Schuster, 1989) and a two-dimensional Ising model, many articles have been written representing biological systems as spin models. In (Baake E., Baake M. and Wagner, 1997) it has been shown that the parallel mutation-selection model can be put in correspondence with the hamiltonian of an Ising quantum chain and in (Saakian and Hu, 2004) the Eigen model of evolution has been mapped into the hamiltonian of one-dimensional quantum spin chains. In this approach the genetic sequence is specified by a sequence of spin values \pm 1 . In more refined models the correspondence is made between the four nucleotides and a set of two binary labels, see (Hermisson, Wagner and Baake M., 2001) for a four-state quantum chain approach. The main 
aim of the works using this approach, see (Baake E., Baake M. and Wagner, 1998), (Wagner, Baake E. and Gerisch, 1998), (Baake E. and Wagner, 2001), (Hermisson, Redner and Wagner, 2002), is to find, in different landscapes, the mean "fitness" and the "biological surplus", in the framework of biological population evolution. As standard assumption, the strength of the mutation is assumed to depend from the distance between two sequences, which is identified with the Hamming distance. We recall that the Hamming distance between two strings of binary labels is given by the number of sites with different labels. Moreover usually it is assumed that the mutation matrix elements are vanishing for Hamming distances larger than 1, i.e. for more than one nucleotide changes. The Hamming distance assumption is clearly unrealistic in the domain of genetic mutations, so the only justification for its use is that this assumption generally allows to solve the problem exactly in the one point mutation scheme or to find more tractable numerical solutions. For example the mutation between the sequence ...GUGU - ACAC . . and the sequences, both differing of one unit, in the Hamming distance, from the original one, ...GUUU - AAAC . . and ...GGGU - ACCC . . implies respectively a change in the free energy, at standard conditions, of $\approx-0.89 \mathrm{kcal} / \mathrm{mol}$ and of $\approx+0.8 \mathrm{kcal} / \mathrm{mol}$, see (SantaLucia, 1998). To assume these transitions equally probable is clearly a rough approximation. Let us note that we use the term transition in a physical general sense. In biology transition is a mutation from a purine (pyrimidine) to a purine (pyrimidine), transversion is a mutation from a purine (pyrimidine) to the other family. So, in the above specified simplified assumption, the transitions have to be really understood as biological transversions. At our knowledge there has been no attempt to apply spin models to obtain the observed equilibrium distribution of oligonucleotides in DNA. Martindale and Konopka (Martindale and Konopka, 1996) have, indeed, remarked that the ranked short (ranging from 3 to 10 nucleotides) oligonucleotide frequencies, in both coding and non-coding region of DNA, follow a Yule distribution. We recall that a Yule distribution (Yule, 1924) is given by

$$
f=a n^{k} b^{n}
$$

where $n$ is the rank and $a, k<0$ and $b$ are 3 real parameters. In order to face this problem, in this paper we propose a spin model where effects of neighbours (not only the nearest ones) and on the whole sequence context is taken into account. To this aim, we build up a quantum and classical spin model in which the strength of the transition matrix does not depend only from the number of different symbols (Hamming distance) between two sequences, but in some sense also from the position of the changed symbols and from the whole distribution of the nucleotides in the sequence. In this paper, we assume that the transition matrix does not vanish only for total spin flip equal \pm 1 , induced by the action of a single step operator, which generally is equivalent to one nucleotide change. Let us recall some phenomenological aspects of mutations. From observations on the characters of spontaneous mutations, it seems possible to point out some common features of almost every studied process. These can be resumed in the following points:

- the mutation rate of a nucleotide depends on nature of its first neighbouring ones;

- mutations occur more frequently in purine/pyrimidine alternating tracts;

- transitions are more frequent than transversions;

- mutations mainly interest dinucleotides CG. 
In modeling mutation mechanism, only paying attemption on the difference between purines and pyrimidines (so that we only consider transversions), we take only into account the first two of the four points above listed.

In a context slightly different, it has been remarked (Frappat, Minichini, Sciarrino and Sorba, 2003) that the rank of codon usage probabilities follows a universal law, that is independent of the biological species, the rank ordered distribution $f(n)$ being nicely fitted by a sum of an exponential part and a linear part. Of course the same codon occupies in general two different positions in the rank distribution function for two different species, but the shape of the function is the same. More specifically, for each biological species, codons are ordered following the decreasing order of the values of their usage probabilities, i.e. the codon with rank $n=1$ corresponds to the one with highest value of the codon usage frequency, codon with rank $n=2$ is the one corresponding to the next highest value of the codon usage frequency, and so on. In that article $f(n)$ was plotted versus the rank and was well fitted by the following function

$$
f(n)=\widehat{\alpha} e^{-\widehat{\eta} n}-\widehat{\beta} n+\widehat{\gamma}
$$

where $0.0187 \leq \widehat{\alpha} \leq 0.0570,0.050 \leq \widehat{\eta} \leq 0.136,0.8210^{-4} \leq \widehat{\beta} \leq 3.6310^{-4}$, depend on the biological species, essentially on the total exonic $G C$ content. The four constants have to satisfy the normalization condition

$$
\sum_{n} f(n)=1
$$

The value of the constant $\widehat{\gamma}=0.0164$ is approximately equal to $1 / 61$, i.e. the value of the codon usage probability in the case of uniform and not biased codon distribution (not taking into account the 3 Stop codons), so really eq.(2) depends on only two free parameters. Therefore the first two terms in eq.(2) can be viewed as the effect of some bias mechanism. We assume that this bias is only the effect of the mutation and selection pressure, which we modelise by the effect of a suitable fitness and a mutation matrix which depend on the change of the labels identifying the codons in the so called crystal basis model of the genetic code, see (Frappat, Sciarrino and Sorba, 1998). The paper is organised in the following way. In Sec. 2 we briefly review the mathematical tools we use, putting in an Appendix, to make the article self-consistent, the basic definitions and properties. We identify a sequence of $N$ nucleotides or a $N$ spins chain as a vector state of an irreducible representation (irrep.) of $U_{q \rightarrow 0}(s l(2))$. Transitions between sequences are introduced in terms of operators connecting vector states belonging or not belonging to the same irrep.. In Sec. 3 we build up a quantum spin model described by a hamiltonian whose diagonal part, in the basis vectors of the irrep., represents the fitness and the off diagonal terms describes the mutations. Let us point out that we do not aim to describe mutations in DNA as quantum effects. We use the quantum mechanics formalism only as a very useful language to introduce the mutations inducing operators. The model, which can appear unphysical if applied to a quantum spin chain, should be considered, on the light of the previous remarks on the application to the biological evolution, as a guideline toward the search of solutions which can reproduce the observed oligonucleotide distribution. In some sense we proceed in the backward direction with respect to the usual approach: we go from the quantum to the classical model. In Sec. 4, using the results of the previous section, we write classical kinetic equations for the probabilities and we solve it numerically, in the case of short oligonucleotide sequences. In Sec. 
5 we discuss our results. In Sec. 6 we extend the model to four letter alphabet, that is we identify the nucleotides with the fundamental 4-dim irreducible representation of $U_{q \rightarrow 0}(\operatorname{sl}(2) \oplus \operatorname{sl}(2))$. In Sec. 7 the four letters model described in Sec. 6 is applied and numerically solved for the codons. The numerical solution of the model gives a stationary configuration for the distribution frequency which is indeed nicely fitted by the function $f(n)$. These solutions, but largely not their shape, depend on the numerical values of the arbitrarily choosen parameters of the mutation matrix and of the fitness. However a choice of the parameters in severe contradiction with the reality, implying, e.g., a ratio of transversions over transitions mutations very high or very low, seems to destroy the goodness of the fit. At the end a few conclusions and future possible developments are presented.

\section{Mutations and Crystal basis}

An ordered sequence of $\mathrm{N}$ nucleotides, characterized only by the purine or pyrimidine character, that is a string of $N$ binary labels or spins, can be represented as a vector state belonging to the $\mathrm{N}$-fold tensor product of the fundamental irriducible representation (irrep.) (labeled by $J=1 / 2$ ) of $\mathcal{U}_{q \rightarrow 0}(s l(2))$ (Kashiwara, 1990), see Appendix A. This parametrization allows to represent, in a simple way, the mutation of a sequence as a transition between vectors, which can be subjected to selection rules and whose strength depends from the two concerned states.

\subsection{Labelling the state}

We identify a N-nucleotide sequence as a state

$$
|\mathbf{J}\rangle=\left|J_{3}, J^{N}, \ldots, J^{2}\right\rangle
$$

where $J^{N}$ labels the irrep. which the state belongs to, $J_{3}$ is the value of the 3rd diagonal generator of $\mathcal{U}_{q \rightarrow 0}(\operatorname{sl}(2))\left(2 J_{3}=n_{R}-n_{Y}, n_{X}\right.$ being the number of $X$ elements in the sequence) and $J^{i}(2 \leq i \leq$ $N-1$ ) are $N-2$ labels needed to remove the degeneracy of the irreps. in the $N$-fold tensor product in order to completely identify the state. These further labels can be seen as the labels identifying the irrep. which the state, corresponding to the sequence truncated to the $i$-th element, belongs to. We introduce a scalar product, such that

$$
\langle\mathbf{J} \mid \mathbf{K}\rangle= \begin{cases}1 & \text { if } J_{3}=K_{3} \text { and } J^{i}=K^{i} \forall i \\ 0 & \text { otherwise }\end{cases}
$$

As an example, we can consider a trinucleotidic string $(N=3)$ and label the eight different spin chains in the following way, using the crystal basis representation $\left|J_{3}, J^{N}, \ldots, J^{2}\right\rangle$ :

$$
\begin{aligned}
\uparrow \downarrow \downarrow & =\left|-\frac{1}{2}, \frac{1}{2}, 0\right\rangle & & \uparrow \downarrow \uparrow=\left|\frac{1}{2}, \frac{1}{2}, 0\right\rangle \\
\downarrow \uparrow \downarrow & =\left|-\frac{1}{2}, \frac{1}{2}, 1\right\rangle & & \uparrow \uparrow \downarrow=\left|\frac{1}{2}, \frac{1}{2}, 1\right\rangle \\
\downarrow \downarrow \downarrow & =\left|-\frac{3}{2}, \frac{3}{2}, 1\right\rangle & & \downarrow \downarrow \uparrow=\left|-\frac{1}{2}, \frac{3}{2}, 1\right\rangle \\
\downarrow \uparrow \uparrow & =\left|\frac{1}{2}, \frac{3}{2}, 1\right\rangle & & \uparrow \uparrow \uparrow=\left|\frac{3}{2}, \frac{3}{2}, 1\right\rangle .
\end{aligned}
$$


In our approach, sequences with the same number of spin up and down, placed in different sites, are described by different states. This has a phenomelogical support; indeed, in the case of RNA sequences, the values of the free energy $(-\Delta G)$, on Kcal $/ \mathrm{mol}$ at standard conditions, for four different sequences made of two $\mathrm{CC}$ and two GG, i.e. two $\mathrm{R}$ and two $\mathrm{Y}$, as reported in Table I of (Xia et al., 1998): CCGG (4.55), GGCC (5.37), CGCG (3.66), GCGC (4.61), are different. At this stage the crystal basis provides, at least. an alternative way of labelling any finite spin sequence, mapping any sequence in a vector state of an irrep., but we know that in physics and mathematics the choice of appropriate variables is of primary importance to face a problem. Indeed we argue that these variables are suitable to partially describe non local events which affect the mutations. We only consider a single spin flip, which in most cases, but not always, is equivalent to a single nucleotide mutation. Flipping one spin can induce a transition to a state belonging or not belonging to the irrep of the original state. From the results of Appendix A we see that to identify a nucleotide sequence as a state of an irrep. requires to fix the number of RY contracted couples occurring in the considered sequence ${ }^{1}$. Therefore, flipping a spin implies or the creation or the deletion of a RY contracted couple, corresponding respectively to a variation of $-1 \mathrm{o}+1$ on the value of the $J^{N}$ and, in case, of some others $J^{i}(2 \leq i \leq N-1)$, or to leave unmodified the number of contracted couples (so that the variation of $J^{N}$ is $\Delta J^{N}=0$, but some other $J^{i}$ may change). In the following we classify the mutations of a single spin flip in N-nucleotide string, according to the induced variation in the string labels $J_{3}, J^{N}, \ldots, J^{2}$. We focus our attention on the spin flip of the $i$-th position, but sometimes the transition will also effect other nucleotides. We call left (right) side free the nucleotides on the left (right) of $i$-th position and not contracted (in the sense expressed in Appendix A) with another one on the same side. Let $R_{l}$ be the initial (before mutation) number of the left side free purines and $Y_{r}$ the initial number of the right side free pyrimidines. We want to count the total number of contracted $R Y$ couples (before and after mutation) in the string, so we call $R_{i n}\left(R_{f i}\right)$ the number, in the initial (final) state, of $R$ preceding some $Y$, which is not on the same side, and not contracted with any $Y$ on their side. In the same way, with $Y_{i n}\left(Y_{f i}\right)$ we refer to the number of $Y$ following some $R$, which is not on the same side, and not contracted with any $R$ on their side. If a $R \rightarrow Y$ mutation $\left(\Delta J_{3}=-1\right)$ occurs in $i$-th position, then $R_{\text {in }}=R_{f i}+1$ and $Y_{\text {in }}=Y_{f i}-1$, where $R_{\text {in }}=R_{l}+1$ and $Y_{i n}=Y_{r}$. We can distinguish different string configurations around the $i$-th position, so that a single nucleotide mutation in $i$-th position can correspond to different variations in the string labels. We have that $\Delta J^{N}=\left|R_{f i}-Y_{f i}\right|-\left|R_{i n}-Y_{i n}\right|$

- If $\mathbf{R}_{\mathbf{l}}=\mathbf{Y}_{\mathbf{r}}$ then $R_{i n}-1=Y_{i n}$, so that $\left|R_{i n}-Y_{i n}\right|=1$; after mutation, $R_{f i}=Y_{f i}-1$, so that $\left|R_{f i}-Y_{f i}\right|=1$. Then the variation of $J^{N}$ is $\Delta J^{N}=0$. We distinguish two subcases:

1. $R_{l}=Y_{r} \neq 0: \Delta J^{2}=0, \ldots, \Delta J^{i-1}=0, \Delta J^{i}=-1, \ldots, \Delta J^{k-1}=-1, \Delta J^{k}=0, \ldots, \Delta J^{N}=$ $0(2 \leq N-1 ; i+1 \leq k \leq N)$;

2. $R_{l}=Y_{r}=0: \Delta J^{i}=0 \forall i$.

- If $\mathbf{R}_{\mathbf{l}}>\mathbf{Y}_{\mathbf{r}}$, i.e. $R_{l}=Y_{r}+g(g>0)$, then $\left|R_{\text {in }}-Y_{i n}\right|=g+1$ and $J^{N}=\frac{1}{2}(g+1)$; after mutation, $\left|R_{f i}-Y_{f i}\right|=g-1$ and $J^{N}=\frac{1}{2}(g-1)$. Then $\Delta J^{N}=-1$. We distinguish two subcases:

\footnotetext{
${ }^{1}$ For readers familiar with physics formalism, contraction should be understood in the same sense of contraction of creation-annihilation operators in the Wick expansion
} 
1. $Y_{r}=0: \Delta J^{2}=0, \ldots, \Delta J^{i-1}=0, \Delta J^{i}=-1, \ldots, \Delta J^{N}=-1(2 \leq i \leq N)$;

2. $Y_{r} \neq 0: \Delta J^{2}=0, \ldots, \Delta J^{i-1}=0, \Delta J^{i}=-1, \ldots, \Delta J^{N}=-1(3 \leq i \leq N-1)$.

- If $\mathbf{R}_{\mathbf{l}}<\mathbf{Y}_{\mathbf{r}}$, i.e. $R_{l}=Y_{r}-g(g>0)$, then $J^{N}=\frac{1}{2}(g-1)$; after mutation, $J^{N}=\frac{1}{2}(g+1)$, so that $\Delta J^{N}=1$. We distinguish two subcases:

1. $R_{l}=0: \Delta J^{2}=0, \ldots, \Delta J^{m-1}=0, \Delta J^{m}=1, \ldots, \Delta J^{N}=1(2 \leq m \leq N, m \neq i)$;

2. $R_{l} \neq 0: \Delta J^{2}=0, \ldots, \Delta J^{i-1}=0, \Delta J^{i}=-1, \ldots, \Delta J^{k-1}=-1, \Delta J^{k}=0, \Delta J_{k+1}=$ $1, \Delta J^{N}=1(2 \leq N-2 ; i+1 \leq k \leq N-1)$.

In the case of mutation $Y \rightarrow R$, for a fixed string configuration, the selection rules are similar, changing \pm 1 with $\mp 1$. Operators which lead to the above transitions can be built by $J_{-}, A_{i}, A_{i, k}$ and their adjoint operators, defined in the following section.

\subsection{Transition operators}

In this section we write the transition part of the hamiltonian, for the different possible initial configurations of the string. We distinguish different string configurations around the $i$-th position, so that a single nucleotide mutation in $i$-th position can correspond to different variations in the string labels. The transitions inducing operators are built by means of $J_{-}, A_{i}, A_{i, k}$ and their adjoint operators, as below defined.

- If $\mathbf{R}_{\mathbf{l}}=\mathbf{Y}_{\mathbf{r}}$. We distinguish two subcases:

1. $R_{l}=Y_{r} \neq 0$

$$
H_{1}=\sum_{i=2}^{N-1} \sum_{k=i+1}^{N} \alpha_{1}^{i k}\left(A_{i, k} J_{-}+J_{+} A_{i, k}^{\dagger}\right)
$$

2. $R_{l}=Y_{r}=0$

$$
H_{2}=\alpha_{2}\left(J_{-}+J_{+}\right)
$$

- If $\mathbf{R}_{\mathbf{l}}>\mathbf{Y}_{\mathbf{r}}$. We distinguish two subcases:

1. $Y_{r}=0$

$$
H_{3}=\sum_{i=2}^{N} \alpha_{3}^{i}\left(A_{i} J_{-}+J_{+} A_{i}^{\dagger}\right)
$$

2. $Y_{r} \neq 0$

$$
H_{4}=\sum_{i=3}^{N-1} \alpha_{4}^{i}\left(A_{i} J_{-}+J_{+} A_{i}^{\dagger}\right)
$$

- If $\mathbf{R}_{\mathbf{l}}<\mathbf{Y}_{\mathbf{r}}$. We distinguish two subcases:

1. $R_{l}=0$

$$
H_{5}=\sum_{m=2}^{N} \alpha_{5}^{m}\left(J_{-} A_{m}^{\dagger}+A_{m} J_{+}\right)
$$


2. $R_{l} \neq 0$

$$
H_{6}=\sum_{i=2}^{N-2} \sum_{k=i+1}^{N-1} \alpha_{6}^{i k}\left(A_{i, k} J_{-} A_{k+1}^{\dagger}+A_{i, k}^{\dagger} A_{k+1} J_{+}\right)
$$

where $J_{+}$and $J_{-}$are the step operators defined by Kashiwara (Kashiwara, 1990), acting on an irreducible representation with highest weight $J^{N}$, i.e. inducing the transitions $\Delta J^{i}=0, \forall i$

$$
\begin{aligned}
A_{i, k}|\mathbf{J}\rangle=\mid & \left.J_{3}, J^{N}, ., J^{k}, J^{k-1}-1, ., J^{i}-1, J^{i-1}, ., J^{2}\right\rangle \\
& (2 \leq i \leq N-1 \quad i+1 \leq k \leq N) \\
A_{i}|\mathbf{J}\rangle= & \left|J_{3}, J^{N}-1, \ldots, J^{i}-1, J^{i-1}, \ldots, J^{2}\right\rangle \\
& (2 \leq i \leq N) \\
B_{m}|\mathbf{J}\rangle= & \left|J_{3}, J^{N}+1, \ldots, J^{m}+1, J^{m-1}, \ldots, J^{2}\right\rangle \\
& (2 \leq m \leq N)
\end{aligned}
$$

Therefore $A_{i, k}^{\dagger}$ is the operator which increase by 1 the value of $J^{l}$, for $k-1 \leq l \leq i$ and $B_{m}=A_{m}^{\dagger}$. Let us remark that in the above equations only the writing order of $A_{k+1}$ and $J_{ \pm}$has to be respected as

$$
\left[A_{k+1}, J_{ \pm}\right] \neq 0
$$

while $(i<k<N)$

$$
\left[A_{i, k}, A_{k+1}\right]=\left[A_{i, k}, J_{ \pm}\right]=0
$$

The following commutation relations can be useful for understanding the action of the transition hamiltonian as well as for further developments:

$$
\begin{gathered}
{\left[A_{i}, J_{3}\right]=\left[A_{i, k}, J_{3}\right]=0 \quad \forall i, k} \\
{\left[A_{i} J_{-}, J_{3}\right]=A_{i} J_{3} \quad\left[J_{+} A_{i}^{\dagger}, J_{3}\right]=-J_{+} A_{i}^{\dagger}} \\
{\left[A_{i, k} J_{-}, J_{3}\right]=A_{i, k} J_{-} \quad\left[J_{+} A_{i, k}^{\dagger}, J_{3}\right]=-J_{+} A_{i, k}} \\
{\left[A_{i, k} J_{-} A_{k+1}^{\dagger}, J_{3}\right]=A_{i, k} J_{-} A_{k+1}^{\dagger}} \\
{\left[A_{i, k}^{\dagger} A_{k+1} J_{+}, J_{3}\right]=-A_{i, k}^{\dagger} A_{k+1} J_{+}}
\end{gathered}
$$

A few words to comment on the above equations. Let us consider a mutation $R \rightarrow Y$ which involve a transition $J^{N}=-1$ (case $R_{l}>Y_{r}$ ); the considered transition also entails $\Delta J_{3}=-1$, so we have to apply the operator $J_{-}$, as well as the operator $A_{i}$. Of course, first we have to lower by 1 the value of $J_{3}$, then to modify $J^{N}$, otherwise the initial state may eventually be annihilated, even if the transition is allowed (in the case $J^{N}-1<J_{3}$ ). Likewise, in corrispondence of a transition 
$Y \rightarrow R\left(\Delta J_{3}=+1\right)$, first the change $J^{N} \rightarrow J^{N}+1$ has to be maked, then $J_{3} \rightarrow J_{3}+1$. To write a self-adjoint operator, we have to add to the operator, which gives rise to the transition $Y \rightarrow R$, the one which leads to $R \rightarrow Y$, leaving the rest of the string unmodified, that is

$$
A_{i} J_{-}+J_{+} A_{i}^{\dagger}
$$

This operator leads to the mutation $Y \rightarrow R$ or $R \rightarrow Y$ for a nucleotide in $i$-th position, in a string with $R_{l}>Y_{r}$. If the mutation $R \rightarrow Y$ rises the value of $J^{N}$, first $J^{N}$ has to be modified, then $J_{3}$; with the aim to write a self adjoint operator, we write

$$
J_{-} A_{m}^{\dagger}+A_{m} J_{+}
$$

The above operator gives rise to mutations $R \rightarrow Y$ and $Y \rightarrow R$ for a nucleotide in $i$-th position, preceding the $m$-th one, in the case $R_{l}=0, Y_{r} \neq 0$. Let us remark that eq.(9) is included in eq.(8), if the coupling constants $\alpha_{4}^{i}$ are assumed equal to $\alpha_{3}^{i}$; in eq.(11), only the writing order for $A_{k+1}$ (and its adjoint) and $J_{ \pm}$has to be respected. Let us also note that when $\Delta J^{N}=0$ there is no need to order the operators.

\section{The quantum spin model}

Assuming now that the coupling constants do not depend on $i, k, m$, we can write the transition hamiltonian $H_{I}$ as

$$
H_{I}=\mu_{1}\left(H_{3}+H_{5}\right)+\mu_{2} H_{1}+\mu_{3} H_{2}+\mu_{4} H_{6}
$$

The total hamiltonian of the model will be written as

$$
H=H_{0}+H_{I}
$$

where $H_{0}$ is the diagonal part in the choosen basis and, in the following, is assumed to be $H_{0}=\mu_{0} J_{3}$. We let the fenomenology suggests us the scale of the values of the coupling constants of $H_{I}$. We want to write an interaction term which makes the mutation in alternating purinic/pyrimidinic tracts less likely than in polypurinic or polypyrimidinic ones. We mean as a single nucleotide mutation in a polypurinic (polypyrimidinic) tract, a mutation inside a string with all nucleotides $R(Y)$, i.e. a highest (lowest) weight state. Such a transition corresponds to the selection rules $\Delta J^{N}=-1, \Delta J_{3}=$ \pm 1 , i.e. a transition generated by the action of $H_{3}$ and $H_{5}$. In the interaction term $H_{I}$, we give them a coupling constant smaller than the others terms. We introduce, for $\Delta J_{3}= \pm 1$, only four different mutation parameters $\mu_{i}(i=1,2,3,4)$, with $\mu_{1}<\mu_{k}, k>1$.

1. $\mu_{1}$ for mutations which change the irrep., $\Delta J^{N}= \pm 1$, and include the spin flip inside an highest or lowest weight vector;

2. $\mu_{2}$ for mutations which do not change the irrep., $\Delta J^{N}=0$, but modifies other values of $J^{k}$, $\Delta J^{k}= \pm 1$

3. $\mu_{3}$ for mutations which do not change the irrep., $\Delta J^{N}=0$, neither the other values of $J^{k}$, $\Delta J^{k}=0,(2 \leq k \leq N-1)$; 
4. $\mu_{4}$ for mutations which change the irrep., $\Delta J^{N}= \pm 1$, but only in a string with $0 \neq R_{l}<Y_{r}$. We do not introduce another parameter, for mutations generated by $H_{4}$, i.e. $i$-th nucleotide mutation in a string with $R_{l}>Y_{r} \neq 0$, not to distinguish, in a polypurinic string, between a mutation in 2-th position and another one inside the string. Let us emphasize once more that the proposed model takes into account, at least partially, the effect on the transition in the $i$-th site of the distribution of all the spins. Presently we consider only the part of the interaction hamiltonian $H_{I}$, which generates transitions corresponding to one spin flip write, but, in analogous way, we could write more complicated transition operators. Let us illustrate, in a simple example, the difference between this scheme and the standard one, based on the hypotesis of transition probability between chains, only depending by their Hamming distance. Let us consider the following string $R R R R R$. By a single flip spin the string goes in one of the following configurations:

$$
\text { 1) } Y R R R R \text { 2) } R Y R R R \text { 3) } R R Y R R \text { 4) } R R R Y R \quad \text { 5) } R R R R Y
$$

In the models based on the Hamming distance, all the transitions are equally probable, as the final strings are all at the same distance from the original one. In the present scheme the 1-rst transition is ruled by the value of $\mu_{3}$, the transition $2-5$ are ruled by $\mu_{1}$.

Let us stress that our scheme is not equivalent to an Ising model with the transition strength depending on the position. To illustrate the difference with a few examples let us consider the transitions $R Y Y R R \rightarrow R Y Y Y R, R Y Y R Y \rightarrow R Y Y Y Y$, both with a flip in the fourth position, the first one ruled by $\mu_{2}$, the second one by $\mu_{1}$. Mutations in different points can be ruled by the same coupling constant: $R Y Y R R \rightarrow R Y Y Y R, R Y R R Y \rightarrow R Y Y R Y$ with a flip, respectively, in the 4 th and 3rd position ruled by $\mu_{2}$. As already said, the main motivation for introducing this quantum model is that it provides the formal and conceptual language to write the transitions, ensuring in the same time, due to the unitary character of the evolution operator, the conservation of the probability. We shall briefly describe in Sec. 6 the outcome of this model, see (Minichini and Sciarrino 2004a) for more details, which has only been reported to make, hopefully, more clear the structure of the classical model of the next section. Let us point out that there are very strong drawback in trying to further pursue the study of the quantum model, for example superposed states, that is linear combinations of sequences, do exist in such models, while only the different sequences have a biophysical interpretation.

\section{The classical model}

In the previous section we have introduced mutation inducing operators based on the change of the global labels $J^{i}$. Using these results as a guide we write a kinetic equations systems in which the non vanishing mutation matrix entries depend on the labels of the connected sequences. We are interested in finding the stationary or equilibrium configuration of the $2^{N}$ different possible sequence. Writing $p_{\mathbf{J}}(t)$ the probability distribution at time $t$ of the sequence identified by the vector $|\mathbf{J}\rangle$, a decoupled version of selection mutation equation, (see (Hofbauer and Sigmund, 1988) for an exhaustive review), for a haploid organism, can be written as

$$
\frac{d}{d t} p_{\mathbf{J}}(t)=p_{\mathbf{J}}(t)\left(R_{\mathbf{J}}-\sum_{\mathbf{K}} R_{\mathbf{K}} p_{\mathbf{K}}(t)\right)+\sum_{\mathbf{K}} M_{\mathbf{J}, \mathbf{K}} p_{\mathbf{K}}(t)
$$


where $R_{\mathbf{K}}$ is the Malthusian fitness of the sequence corresponding to the vector $|\mathbf{K}\rangle$ and $M_{\mathbf{J}, \mathbf{K}}$ are the entries of a mutation matrix $M$ which satisfies

$$
M_{\mathbf{J}, \mathbf{J}}=-\sum_{\mathbf{K} \neq \mathbf{J}} M_{\mathbf{J}, \mathbf{K}}
$$

The equation (27) is reduced to

$$
\frac{d}{d t} x_{\mathbf{J}}(t)=\sum_{\mathbf{K}}(H+M)_{\mathbf{J}, \mathbf{K}} x_{\mathbf{K}}(t)
$$

where

$$
x_{\mathbf{J}}(t)=p_{\mathbf{J}}(t) \exp \left(\sum_{\mathbf{K}} R_{\mathbf{K}} \int_{0}^{t} p_{\mathbf{K}}(\tau) d \tau\right)
$$

and $H$ is a diagonal matrix, with fitness as entries $\left(R_{\mathbf{K}}=H_{\mathbf{K}, \mathbf{K}}\right)$. In our model the mutation matrix is written as the sum of the partial mutation matrices $M_{i}$ which are obtained by the interaction hamiltonians $H_{i}$ replacing the adjoint operators by the transposed (denoted by an upper labe ${ }^{T}$ ). Assuming now that the coupling constants do not depend on $i, k, m$, we can write the mutation matrix $M$ as (Minichini and Sciarrino, 2004b)

$$
M=\mu_{1}\left(M_{3}+M_{5}\right)+\mu_{2} M_{1}+\mu_{3} M_{2}+\mu_{4} M_{6}+M_{D}
$$

where $M_{D}$ is the diagonal part of the mutation matrix defined by eq.(28). The hierarchy of the values of the coupling constants is fixed as in the previous section.

\section{Results}

The evolution equation of the model for the probabilities will be written in terms of the matrix $\bar{H}=H+M+\lambda \mathbf{1}$, where the fitness can be $H=J_{3}$ (purely additive fitness) and $\lambda$ is choosen in such a way to guarantee $\bar{H}$ is positive. Being $H+M$ irreducible, the composition of equilibrium population is given by

$$
p_{\mathbf{J}}=\frac{\tilde{x}_{\mathbf{J}}}{\sum_{\mathbf{K}} \tilde{x}_{\mathbf{K}}}
$$

where $\tilde{x}_{\mathbf{J}}$ is the Perron-Frobenius eigenvector, see, e.g. (Encyclopedic Dictionary of Mathematics, 1960) of $\bar{H}$. In (Minichini and Sciarrino, 2004b) the numerical solutions of the model have been reported, with a suitable choice of the value of the parameters, for $\mathrm{N}=3,4,6$. Before discussing these results, we point out explicitly the main features of our model. $M$ describes an interaction on the $i$-th spin neither depending on the position nor on the nature of the closest neighbours, but which takes into account, at least partially, the effects, on the transition in the $i$-th site, of the distribution of all the spins, that is non local effects. Indeed it depends on the "ordered" spin orientation surplus on the left and on the right of the $i$-th position. Should it not depend on the order, it may be considered as a mean-field like effect. Moreover $\Delta J_{3}= \pm 1$ transitions are allowed, which, e.g. for $\mathrm{N}=4$, can be considered or as the flip of a spin combined with an exchange of the two, oppositely oriented, previous or following spins or as the collective flip of particular three spin systems, containing a two spin system with opposite spin orientations (see example below). Biologically, the transition depends 
in some way on the "ordered" purine surplus on the left and on the right of the mutant position. Let us briefly comment on the physical-biological meaning of the "ordered" spin sequence. Our aim is to study finite oligonucleotide sequence in which a beginning and an end are defined. This implies we can neither make a thermodynamic limit on $N$ nor define periodic conditions on the spin chain. So we have to take into account the "edge" or "boundary" conditions on the finite sequence. An analogous problem appears in determing thermodynamic properties of short oligomers and, in this framework, in (Goldstein and Benight, 1992) the concept of fictitious nucleotide pairs E and E' has been introduced, in order to mimick the edge effects. The ordered couple of RY takes into account in some way the different interactions of $\mathrm{R}$ and $\mathrm{Y}$ with the edges. For example, the transition matrix, on the above basis (for $\mathrm{N}=3$ ) is the following one, up to a multiplicative dimensional factor $\mu_{0}$

$$
M=\left(\begin{array}{llllllll}
x & \delta & 0 & \gamma & \epsilon & 0 & \epsilon & 0 \\
\delta & x & 0 & 0 & 0 & \epsilon & 0 & \epsilon \\
0 & 0 & x & \delta & \epsilon & 0 & \epsilon & 0 \\
\gamma & 0 & \delta & x & 0 & \epsilon & 0 & \epsilon \\
\epsilon & 0 & \epsilon & 0 & x & \delta & 0 & 0 \\
0 & \epsilon & 0 & \epsilon & \delta & x & \delta & 0 \\
\epsilon & 0 & \epsilon & 0 & 0 & \delta & x & \delta \\
0 & \epsilon & 0 & \epsilon & 0 & 0 & \delta & x
\end{array}\right)
$$

where the diagonal entries $x$, not explicitly written, are given by eq.(28). Note that the above matrix depends only on three coupling constants due to the very short length of the chain. For $N \geq 4$ the 4th coupling constant (denoted in the following by $\eta$ ) will appear. Let us emphasize that the mutation matrix $M($ (33) $)$ does not only connect states at unitary Hamming distance. As an example, we write explicitly the transitions from $\left|\frac{1}{2}, \frac{1}{2}, 0\right\rangle(\uparrow \downarrow \uparrow)$ and from $\left|-\frac{1}{2}, \frac{1}{2}, 0\right\rangle(\uparrow \downarrow \downarrow)$
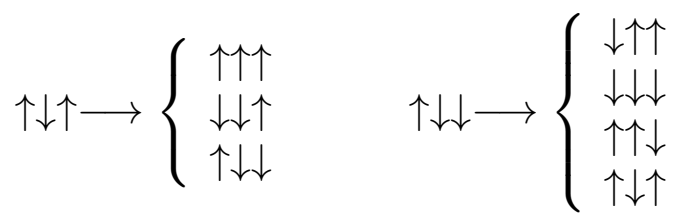

The first transition of the second example can be regarded as a spin-flip of the three spins. Let we explicitly write, for $N=3$, the mutation matrix, which allows transitions only between chains at Hamming distance equal to one, with coupling constant $\alpha$.

$$
H_{\mathrm{Hamm}}=\left(\begin{array}{cccccccc}
y & \alpha & 0 & \alpha & \alpha & 0 & 0 & 0 \\
\alpha & y & 0 & 0 & 0 & \alpha & 0 & \alpha \\
0 & 0 & y & \alpha & \alpha & 0 & \alpha & 0 \\
\alpha & 0 & \alpha & y & 0 & 0 & 0 & \alpha \\
\alpha & 0 & \alpha & 0 & y & \alpha & 0 & 0 \\
0 & \alpha & 0 & 0 & \alpha & y & \alpha & 0 \\
0 & 0 & \alpha & 0 & 0 & \alpha & y & \alpha \\
0 & \alpha & 0 & \alpha & 0 & 0 & \alpha & y
\end{array}\right)
$$

where the diagonal entries, not explicitly written, are given by eq.(28). Note that, even if we put in eq.(33) all the constant equal to $\alpha(\delta=\gamma=\varepsilon=\alpha)$, we do not get the Hamming hamiltonian (34). If we order (in a decreasing way) the equilibrium probabilities, we obtain, using the mutation matrix with Hamming distance, a rank ordered distribution of transition probability like that in fig 1 for 
$N=4$. Its shape does not depend on the value of $\alpha$. The rank-ordered distribution of the probabilities shows a plateaux structure: every plateaux contains spin sequences at the same Hamming distance from the sequence with the highest value of the fitness. Using the mutation matrix (133), the rank ordered probabilities distribution does not show a plateaux structure, but its shape is well fitted by a Yule distribution (fig 2), like the observed frequency distribution of oligonucleotidic in the strings of nucleic acids (Martindale and Konopka, 1996). Let us observe that we obtain a Yule distribution (and not a plateaux structure) even if all parameters in (33) are tuned at the same value, which means that the distribution is the outcome of the model and not of the choice of the values of the coupling constants. Analogous resultes are obtained for $N=6$ (fig [3). Let us point out that:

i) our model is not equivalent to a model where the intensity depends on the site undergoing the transition, or from the nature of the closest neighbours or the number of the $R$ and $Y$ labels of the sequence; indeed essentially the intensity depends on distribution in the sequence of the $R$ and $Y$;

ii) the ranked distribution of the probabilities follows a Yule distribution law, but as the value of the parameter $\mathrm{b}$ is close to the unity, the distribution is equally well fitted by a Zipf law (Zipf, 1949) $\left(f=a n^{k}\right)$, in agreement with the remark of (Martindale and Konopka, 1996).

Let us also briefly recall the outcomes of the genetically inspired quantum spin model presented in Sec. 3. We can study the time evolution of an initial state, representing a given spin chain, and evaluate the probability of transition in another one, if $H$ is the hamiltonian which generates the dynamics of the system. The matrix form of $H$, on the above basis for a fixed initial state, is obtained (for $N=3$ ), by replacing in eq.(33) the diagonal terms by the eigenvalues of $J_{3}$, i.e. by, respectively, $(-1,1,-1,1,-3,-1,1,3)$ (up a multiplicative factore 1/2). Analogously we can study the dynamics of an ordered quantum spin chain, with an interaction Hamiltonian, leading to transitions with the same probability between nucleotide strings at unit Hamming distance, whose matrix, for $N=3$, is obtained by eq.(34) by replacing the diagonal terms with the eigenvalues of $J_{3}$. In order to evaluate the probabilities of transition, we cannot analytically study the time evolution of an initial state, representing a fixed spin sequence, as ruled by eq.(33) with the change of the diagonal terms, but we can find a numerical solution. The transition probability between two states, belonging to the crystal basis, exhibits the quantum mechanically typical oscillating behaviour as a function of the time. We define a time-averaged transition probability (initial state $(\mathrm{i}) \longrightarrow$ final state $(\mathrm{f})$ )

$$
<p_{i f}>=\frac{1}{T} \int_{0}^{T} p_{i f}(t) d t
$$

where the value of $T$ will be numerically fixed to a value, such that the r.h.s. of eq.(35) becomes stable. If we order (in a decreasing way) the average transition probability from an initial state to every other chain, if (34) is the hamiltonian, we obtain a rank ordered distribution of transition probability like that in fig 1. Its shape does not depend by the choice of initial state or by the coupling constant $\alpha$ value. We always get the same structure, for models with transition probability only depending on Hamming distances. So the rank-ordered distribution of the average transition probability shows a plateaux structure: every step contains spin chains at the same Hamming distance from the initial one. In the case of the model which we propose here, i.e. the hamiltonian in (33), which we call crystal basis model, the distribution of rank ordered average transition probability does not show a plateaux structure, but its shape is well fitted by a Yule distribution like that in fig.2. Also in the 
quantum model, we obtain a Yule distribution (and not a plateaux structure) even if all parameters in (33) are tuned at the same value. In this case, the state, labelled by 1 in the plots, is the initial one. The ranked distribution of the probabilities, not averaged in time, computed for several values of the time, also follows generally a Yule distribution law. Moreover we still remark that, for the highest value of $N$, the distribution is equally well fitted by a Zipf law, i.e. $b=1$ in eq.(1), but not for the lowest values of $N$, in agreement with the remark of (Martindale and Konopka, 1996.

\section{The four letter model}

In order to label a sequence of $\mathrm{N}$ nucleotides, taking into account that they belong to the four letter set $\{\mathrm{C}, \mathrm{T} / \mathrm{U}, \mathrm{G}, \mathrm{A}\}$, we assign the 4 nucleotides to the 4-dim irreducible fundamental representation (irreps.) $(1 / 2,1 / 2)$ of $\mathcal{U}_{q \rightarrow 0}(\operatorname{sl}(2) \oplus \operatorname{sl}(2))$ (Frappat, Sciarrino and Sorba, 1998) with the following assignment for the values of the third component of $\vec{J}$ for the two $\operatorname{sl}(2)$ which in the following will be denoted as $s l_{H}(2)$ and $s l_{V}(2)$ :

$$
\mathrm{C} \equiv\left(+\frac{1}{2},+\frac{1}{2}\right) \quad \mathrm{T} / \mathrm{U} \equiv\left(-\frac{1}{2},+\frac{1}{2}\right) \quad \mathrm{G} \equiv\left(+\frac{1}{2},-\frac{1}{2}\right) \quad \mathrm{A} \equiv\left(-\frac{1}{2},-\frac{1}{2}\right)
$$

It follows that an ordered sequence of $\mathrm{N}$ nucleotides can be represented as a vector belonging to the $\mathrm{N}$-fold tensor product of the fundamental irriducible representation of $\mathcal{U}_{q \rightarrow 0}(\operatorname{sl}(2) \oplus \operatorname{sl}(2))$, in a straightforward generalization of the approach followe in Sec.2 for $\mathcal{U}_{q \rightarrow 0}(\operatorname{sl}(2))$. In the following we use the symbols $X$ for $\mathrm{C}, \mathrm{G}$ and $Z$ for U,A. In the formalism of $\mathcal{U}_{q \rightarrow 0}(\operatorname{sl}(2) \oplus \operatorname{sl}(2))$ all the previous results have to be understood to refer to $s l_{V}(2)$. Now we identify a N-nucleotide sequence as a state

$$
\left|\mathbf{J}_{H} \mathbf{J}_{V}\right\rangle=\left|J_{3, H}, J_{3, V} ; J_{H}^{N}, J_{V}^{N} ; \ldots ; J_{H}^{2}, J_{V}^{2}\right\rangle
$$

where $J_{m}^{N}(m=H, V)$ labels the irrep. which the state belongs to, $J_{3, m}$ is the value of the $3 \mathrm{rd}$ diagonal generator of $\mathcal{U}_{q \rightarrow 0}\left(s l_{m}(2)\right)\left(2 J_{3, H}=n_{X}-n_{Z}, 2 J_{3, V}=n_{R}-n_{Y}\right)$ and $J_{m}^{i}(2 \leq i \leq N-1)$ are $2(N-2)$ labels needed to completely identify the state. As an example, the trinucleotidic string CGA is labeled by

$$
|C G A\rangle=\left|\left(\frac{1}{2}\right)_{H},-\left(\frac{1}{2}\right)_{V} ;\left(\frac{1}{2}\right)_{H},\left(\frac{1}{2}\right)_{V} ;(1)_{H}(1)_{V}\right\rangle
$$

The previously introduced scalar product is straightforwardly generalized. In the present paper, we only consider a single spin flip in $\mathrm{H}$ or $\mathrm{V}$ spin or in both $\mathrm{H}$ and $\mathrm{V}$, which in most cases, but not always, is equivalent to a single nucleotide mutation. Obviously a $\mathrm{H}$ spin flip (V and H,V flip) corresponds, respectively, to a biological transition (transversion). Flipping one spin can induce a transition to a state belonging or not belonging to the irrep. of the original state. From an immediate generalisation of the results of Appendix A, we need, to identify a nucleotide sequence as a state of an irrep., to fix the number of RY and XZ contracted couples occurring in the considered sequence. Therefore flipping a spin implies or the creation or the deletion of a RY or XZ or both contracted couple, corresponding, respectively, to a variation of -1 o +1 on the value of the $J_{V}^{N}, J_{H}^{N}$ or both and, possibly, of some others $J_{m}^{i}(2 \leq i \leq N-1)$, or to leave unmodified the number of contracted couples (so that $\Delta J_{m}^{N}=0$, but some other $J_{m}^{i}$ are modified). We focus our attention on the spins flip of the $i$-th position and we go on in a completely analogous way as in Sec. 2, but taking into 
account the two couples RY and XZ. Assuming, as previously, that the coupling constants do not depend on $i, k, m$, we write the mutation matrix $M$ as

$$
\begin{aligned}
M & =M_{H}+M_{V} \\
& =\mu_{1}\left(M_{3, H}+M_{5, H}\right)+\mu_{2} M_{1, H}+\mu_{3} M_{2, H}+\mu_{4} M_{6, H} \\
& +\lambda_{1}\left(M_{3, V}+M_{5, V}\right)+\lambda_{2} M_{1, V}+\lambda_{3} M_{2, V}+\lambda_{4} M_{6, V}+M_{D}
\end{aligned}
$$

where $M_{D}$ is the diagonal part of the mutation matrix defined by eq.(28), and $M_{k, m}(k=1,2,3,5,6 ; m=$ $H, V)$ are the off-diagonal mutation matrices defined by the following operators, where we have omitted to explicitly write the coupling constants

$$
\begin{gathered}
H_{1, m}=A_{i, k ; m} J_{-, m}+J_{+, m} A_{i, k ; m}^{\dagger} \\
H_{2, m}=J_{-, m}+J_{+, m} \\
H_{3, m}=A_{i ; m} J_{-, m}+J_{+, m} A_{i ; m}^{\dagger} \\
H_{4, m}=A_{i ; m} J_{-, m}+J_{+, m} A_{i ; m}^{\dagger} \\
H_{5, m}=J_{-, m} A_{m ; m}^{\dagger}+A_{m ; m} J_{+, m} \\
\left.H_{6, m}=A_{i, k ; m} J_{-, m} A_{k+1 ; m}^{\dagger}+A_{i, k ; m}^{\dagger} A_{k+1 ; m} J_{+, m}\right)
\end{gathered}
$$

Note that in eq.(39) we have not introduced a coupling term between the two $s l(2)$, i.e. a mutation matrix of the type $M_{H, V} \propto J_{+, H} J_{+, V}$ or $M_{H, V} \propto J_{-, H} J_{-, V}$. In order to fit the phenomenological observation that the transitions occur more frequently than the transversions, we have to fix the coupling constants $\lambda$ of the order of $1 / 2-1 / 3$ of the coupling constants $\mu$. Let us remark that, with the chosen mutation matrix eq.(39), a single spin mutation does not correspond necessarily to a $\mathrm{H}$-spin or $\mathrm{V}$-spin flip. Indeed the mutations $C \leftrightarrow A$ amd $T \leftrightarrow G$ imply a flip of both the $\mathrm{H}$ and $\mathrm{V}$ spins, therefore these mutations should be depressed.

\section{The rank ordered distribution of codons}

In (Frappat, Sciarrino and Sorba, 1998) a mathematical model, called crystal basis model, for the genetic code has been proposed where from the assignment eq. (36) of the four nucleotides to the 4-dim fundamental $\left(\frac{1}{2}, \frac{1}{2}\right)$ irreducible representation of the quantum group $\mathcal{U}_{q \rightarrow 0}(\operatorname{sl}(2) \oplus \operatorname{sl}(2))$, the codons (3-nucleotide sequence) appear as composite state in the3-fold tensor product of $\left(\frac{1}{2}, \frac{1}{2}\right)$. From the general formalism of the previous section, a codon is identified as a state

$$
\left|\mathbf{J}_{H}\right\rangle \otimes\left|\mathbf{J}_{V}\right\rangle \equiv\left|\mathbf{J}_{H} \mathbf{J}_{V}\right\rangle=\left|J_{3, H}, J_{3, V} ; J_{H}^{3} J_{V}^{3} ; J_{H}^{2}, J_{V}^{2}\right\rangle
$$


For example we have, see (Frappat, Sciarrino and Sorba, 2001) for a list of all the states:

$$
|C G A\rangle=\left|\left(\frac{1}{2}\right)_{H},-\left(\frac{1}{2}\right)_{V} ;\left(\frac{1}{2}\right)_{H},\left(\frac{1}{2}\right)_{V} ;(1)_{H}(1)_{V}\right\rangle
$$

The mutation matrix eq. (39) now becomes

$$
\begin{aligned}
M & =\sum_{m=H, V} \sum_{i=2,3} \mu_{1, m}\left[\left(A_{i, m} J_{-, m}+J_{+, m} A_{i, m}^{T}\right)\right. \\
& \left.+\left(J_{-, m} A_{i, m}^{T}+A_{i, m} J_{+, m}\right)\right]+\mu_{2, m}\left(J_{-, m}+J_{+, m}\right) \\
& +\mu_{3, m}\left(B_{m} J_{-, m}+J_{+, m} B_{m}^{T}\right)+M_{D, m}
\end{aligned}
$$

where

$$
\begin{aligned}
B_{m}|\mathbf{J}\rangle & =\left|J_{3, m}, J_{m}^{3}, J_{m}^{2}-1\right\rangle \\
A_{i, m}|\mathbf{J}\rangle & =\left|J_{3, m}, J_{m}^{3}-1, \ldots J_{m}^{i}-1, \ldots\right\rangle \quad(2 \leq i \leq 3)
\end{aligned}
$$

and $M_{D}$ is the diagonal part of the mutation matrix. We are interested in finding the stationary configuration solution of the eq.(27) for the 64 different possible sequences. We choose the following form for the (purely additive) fitness $H=J_{3, H}+J_{3, V}+\lambda \mathbf{1}, \lambda>0$ ensuring $H+M$ to be positive. Below we report several representative figures in which the obtained numerical solutions are fitted with a function given by eq.(2) (we omit the hat on the parameters). In figg 44, with a suitable choice of the values of the parameters, our results are well fitted. In fig [6 we report another solution where the ratio, denoted by $(H / V)$, between the mutation intensity between transitions and transversions, is chosen larger than one, but the value of the coupling constants do not satisfy the hierarchy $\mu_{1, H}<\mu_{2, H}, \mu_{3, H}$, which is less well fitted. In fig[ $[7$ we report another solution with a unrealistic choice of the values of the parameters of the ratio $(H / V)((H / V) \approx 10)$, which is, indeed, badly fitted by a function given by eq.(21). Finally in fig 8 we report another solution, also badly fitted, where $(H / V) \approx 10^{-1}$. This last result is a consequence of the fact that we have chosen a fitness symmetric for the exchange $H \leftrightarrow V$. Therefore the exchange of the values of the coupling constants between $M_{H}$ and $M_{V}$ gives the same shape of the distribution. Of course the rank of the same codon is, in general, different in the two cases. Summarizing, we can state that the numerical solutions of our model, for arbitrary choice of the values of the coupling constants, are rather well fitted by a function of the type given in eq.(2), with a suitabe choice of the parameters, but that a non realistic choice of the values of the coupling constants, e.g. a ratio of transversion/transition mutation very high or very low, seems to destroy the goodness of the fit. Moreover, it is quite surprising to remark that the values of the parameters in the function eq.(22), which fits our numerical solutions, are of the same order of magnitude of the parameters (depending on the total $G C$ content) found in (Frappat, Minichini, Sciarrino and Sorba, 2003) to best fit the observed rank ordered distribution. In the present paper, the values of $\widehat{\alpha}$ and $\widehat{\eta}$ are found to be slightly larger than the ones computed in (Frappat, Minichini, Sciarrino and Sorba, 2003). Let us stress once more that a mutation matrix $M$, with non diagonal non vanishing entries connecting only codons with Hamming distance equal to one, is unable to reproduce the observed rank ordered distribution as it induces mutation between classes of codons at the same Hamming distance. We have considered separately the finess and mutation matrix for the horizontal and vertical labels of the codons. As, a priori, one can consider also a coupling term 
between the two parts, our simplified treatment has to be considered as a first step in the way of constructing a realistic model. We have also performed a preliminary analysis with a a value, $\rho_{H}$, of the horizontal fitness different from the value, $\rho_{V}$ of the vertical one. It appears that the outcome depends on the ratio $\rho_{H} / \rho_{V}$ as well as on the ratio between the values of the $\rho$ and the value of the transition coupling constant $\mu$ (beside the discussed dependence on the ratio of $\mu_{H} / \mu_{V}$ and on the hierarchy of the values of the differents $\mu_{H}$ and $\mu_{V}$ ). So we believe that a better understanding of the form of the fitness and of the hierarchy of the values of the mutation parameters, as well as on the reliability of a model which explains the rank ordered distribution of the codons as a consequence of the mutation-selection of 64 triplets, is necessary before further pursuing the numerical analysis .

\section{Conclusions}

We have proposed a model not analytically soluble, but which admits an easy numerical solution for short spin chains. Let us emphasize that the main purpose of the proposed scheme is to take into account, at least partially, the effects of the neighbours in the mutation. We point out, once more, that our model is not equivalent to a model where the intensity depends on the site undergoing the transition, or on the nature of the closest neighbours or on the number of the $R$ and $Y$ labels of the sequence; indeed essentially the intensity depends on the distribution in the sequence of the $R$ and $Y$. We find that the numerically computed stationary distribution for short oligonucleotides to follow a Yule o Zipf law, in agreement with the observed distribution. We are far from claiming, for several obious reasons, that our simple model is the only model able to explain the observed oligonucleotide distribution, but that the standard approach using the Hamming distance does not provide such a solution. One may correctly argue that the comparison between the Hamming model, depending on only one parameter and taking into account only one site spin flip, with our model, which depends on four parameters and takes into account spin flip of more than one site, is not meaningful. So we have computed the stationary distribution with a mutation matrix not vanishing for Hamming distance larger than one and allowing the same number of mutations as our model. The result reported in fig 9 shows that the plateaux structure is always the dominant feature. Let us comment on the non point mutations which naturally are present in our model. In literature there is an increasing number of papers that, on the basis of more accurate data, question both the assumptions that mutations occur as single nucleotide and as independent point event. In a quite recent paper (Whelan and Goldman, 2004) have presented a model allowing for single-nucleotide, doublet and triplet mutation, finding that the model provides statistically significant improvements in fits with protein coding sequences. We note that the triplet mutations, for which there is no known inducing mechanism, but which can possibly be explained by large scale event, called sequence inversion in (Whelan and Goldman, 2004), are indeed the kind of mutations, above discussed, that our model naturally describes. Doublet mutations do not appear, due to the assumed spin flip equal \pm 1 , but on one side some of these mutations are hidden by the binary approximation, and on the other side the parameter ruling such mutations, as computed in (Whelan and Goldman, 2004), is lower than the one ruling the triplet mutation. In conclusion the Hamming distance does not seem a suitable measure of the distance in the space of the biological sequences, the crystal basis, on the contrary, seems a better candidate to parametrize the elements of such space. Our model makes use of this 
parametrisation, allows to modelise some non point mutations and exhibits intriguing and interesting features, hinting in the right direction, worthwhile to be further investigated. In the present simple version, the model depends only on 4 (8) parameters in the two letter (resp. four letter) alphabet for any $\mathrm{N}$, which are, very likely, not enough to describe sequences longer that the considered ones. However the model is rather flexible: as shown in the case of the codons, it is easily generalised to the four letter alphabet; besides the obvious introduction of more coupling constants, it allows, e.g., to analyse part of the sequences containing hot spots in the mutation, to take into account doublet mutations (indeed the operator eq.(12) or $A_{i, i+1}^{T}$ describes a doublet spin flip at position i,i+1). Although the very short chain, which we were interested in, can be studied numerically without any use of the crystal basis, we propose a general algorithm, which can be applied to chains of arbitrary length and which can be easily implemented in computers. It is worthwhile to remark that we are trying to compare theoretical results, deriving from simple models, to really observed data, coming from the extremely complex biological world. In this context the crystal basis provides a compact and useful notation to describe the "kinematical" variables which are changed by the dynamics. The generalisation of our approach to the a four letters alphabet, which is easily done replacing $\mathcal{U}_{q}(s l(2))$ by $\mathcal{U}_{q}(s l(2) \oplus \operatorname{sl}(2))$, has been presented and applied to the study of the mutations of the codons. As expected, calculations are more complicated and only a few results in the simple case of the triplets are given. In this framework, further investigation deserve attention, in particular to study oligonucleotide distribution in the four letters alphabet and mutations in long sequences. In conclusion we point out that:

- the crystal basis provides an alternative way of labelling nucleotide sequences, in particular codons or genes, mapping any finite ordered nucleotide sequence in a vector state of an irrep.. We point out that the choice of the limit $q \rightarrow 0$ (crystal basis) is essential for the above identification as, only in this limit, due to Kashiwara theorem (Kashiwara, 1990), the composite states are pure states.

- the mutation matrix $M$ in our model describes an interaction on the $i$-th nucleotide depending on the input-output sequences and, in the flip of one spin (or double spin), inherently takes into account non local effects. So the crystal basis variables are suitable to partially describe non local events which affect the mutations.

- models based on the crystal basis seem, in the light of the obtained results, better candidates than models based on Hamming distance to describe mutations.

As final remark, this article should be seen as a first, simplified attempt to build models, more realistic than the ones based on the Hamming distance, to describe the effects of the mutationselection on the observed distribution of oligonucleotides.

\section{A Appendix A}

Label of $\mathbf{N}$ binary string. Let us recall that the algebra $\mathcal{U}_{q}(\operatorname{sl}(2))$ is defined as a suitable completion of the algebra of polynomes in the generators $\widetilde{J}_{+}, \widetilde{J}_{-}$and $\widetilde{J}_{3}$ (in particular adding the exponential 
series), subject to the following commutation relations:

$$
\begin{aligned}
{\left[\widetilde{J}_{+}, \widetilde{J}_{-}\right] } & =\left[2 \widetilde{J}_{3}\right]_{q} \\
{\left[\widetilde{J}_{3}, \widetilde{J}_{ \pm}\right] } & = \pm \widetilde{J}_{ \pm}
\end{aligned}
$$

where

$$
[x]_{q}=\frac{q^{x}-q^{-x}}{q-q^{-1}}
$$

Moreover some more axioms have to be fulfilled, which endows $\mathcal{U}_{q}(s l(2))$ with a Hopf algebra structure. The vector spaces of the irreducible representations of this algebra are labelled, for $q$ different of root of unity, by a non negative integer or half-integer number $j$ and are of dimension $(2 j+1)$, the basis vectors being denoted by $\psi_{j m},-j \leq m \leq j$. In the limit $q \rightarrow 1$ one recovers the usual $\operatorname{sl}(2)$. Strictly speaking, in the limit $q \rightarrow 0$ the generators are ill defined, but it is possible, see (Kashiwara, 1990), to define new generators $J_{ \pm}, J_{3}\left(=\widetilde{J}_{3}\right)$, whose action on the vector basis of the representation space, still labelled by a non negative integer or half-integer number $j$ and of dimension $(2 j+1)$, is well defined:

$$
J_{3} \psi_{j m}=m \psi_{j m} \quad J_{ \pm} \psi_{j m}=\psi_{j, m \pm 1} \quad J_{ \pm} \psi_{j, \pm j}=0
$$

This special basis in the limit $q \rightarrow 0$ is called a crystal base. Note that the action of $J_{ \pm}$on $\psi_{j m}$ is equal to $\psi_{j, m \pm 1}$ (i.e. the coefficient is always 1 ), contrary to the $\operatorname{sl}(2)$ or $\mathcal{U}_{q}(s l(2))$ case where this coefficient is a complicated function of $j$ and $m$.

It is possible also to define an operator $C$ called Casimir operator (Frappat, Sciarrino and Sorba, 1998) such that:

$$
C \psi_{j m}=j(j+1) \psi_{j m} \Longrightarrow\left[C, J_{ \pm}\right]=\left[C, J_{3}\right]=0
$$

Its explicit expression is given by

$$
C=\left(J_{3}\right)^{2}+\frac{1}{2} \sum_{n \in \mathbb{Z}_{+}} \sum_{k=0}^{n}\left(J_{-}\right)^{n-k}\left(J_{+}\right)^{n}\left(J_{-}\right)^{k}
$$

In (Kashiwara, 1990) it has been shown that the tensor product of two crystal bases labelled by $j_{1}$ and $j_{2}$ can be decomposed into a direct sum of crystal bases labelled, as in the case of the tensor product of two $\operatorname{sl}(2)$ or of $\mathcal{U}_{q}(\operatorname{sl}(2))$ irreducible representations, by an integer or half-integer number $j$ such that

$$
\left|j_{1}-j_{2}\right| \leq j \leq j_{1}+j_{2}
$$

The new peculiar and crucial feature is that now the basis vectors of the $j$-space are pure states, that is they are the product of a state belonging to the $j_{1}$-space and of a state belonging to the $j_{2}$-space, while in the case of $s l(2)$ or of $\mathcal{U}_{q}(s l(2))$ they are linear combinations with coefficients called respectively Clebsch-Gordan coefficients or $q$-Clebsch-Gordan coefficients. Making use of this property any string of $N$ binary label (spin) $x \in\{ \pm=R, Y\}$ can be seen as a state of an irreducible representation (irrep.) contained in $N$-fold tensor product of the the 2-dim fundamental irrep. (labelled by $j=1 / 2$ ) of $s l_{q \rightarrow 0}(2)$ whose state are labelled by $j_{3}= \pm 1 / 2= \pm=C, U$. Therefore, in the most general case, it can be identified by the following $N$ labels 
1. the value $J^{N}$ labelling the irrep. which the state belongs to. This value is computed taking away the $Y$ elements, which are at right of $R$, contracting each of them with a $R$ element on the left, and then summing the numbers of left $R$ and of left $Y$, which are, respectively, at the right of $Y$ and at the left of $R$. In other words this value is computed deleting all ordered couples $R Y$ (of first neighbours) in the sequence and iterating this procedure, on the generated sequence, up to no $Y$ are on the right of any $R$. We refer to the elements which are deleted in the procedure as contracted.

2. the value of $J_{3}$, with $2 J_{3}=n_{C}-n_{U}, n_{x}$ being the number of $x$ elements in the strings

3. the $N-2$ labels $J^{i}(2 \leq i \leq N-1)$, respectively identifying the irrep., which the sequence truncated to the $i$-th element belongs to.

E.g. the $N=5$ string $C C U C U$ is labelled by: $J^{5}=1 / 2, J_{3}=1 / 2, J^{4}=1, J^{3}=1 / 2, J^{2}=1$; the string $C C C C C$ is labelled by: $J^{5}=5 / 2, J_{3}=5 / 2, J^{4}=1, J^{3}=1 / 2, J^{2}=1$; the string $C U U C C$ is labelled by: $J^{5}=3 / 2, J_{3}=1 / 2, J^{4}=1, J^{3}=1 / 2, J^{2}=0$.

Multiplicity of $\operatorname{sl}(2)$ irreps. in the tensor product. It is useful to know the number (multiplicity) of irreps. labelled by the same value of $J$ appearing in the $N$-fold tensor product of the fundamental representanio $j=1 / 2$, i.e. the coefficients $m_{i}$ appearing in the identity

$$
\otimes^{N} \mathbf{1} / \mathbf{2}=\otimes^{N} J^{(1)}=J^{(N)} \oplus \sum_{k \geq 1} m_{N-2 k}^{N} J^{(N-2 k)}
$$

The number $m_{N-2 k}^{N}$, giving the multiplicity of the irrep. $J^{(i)}$ in the tensor product is given by (Kirillov, 1991)

$$
m_{N-2 k}^{N}=\left[\left(\begin{array}{c}
m \\
k
\end{array}\right)-\left(\begin{array}{c}
m \\
k-1
\end{array}\right)\right]
$$

One gets:

$$
\begin{gathered}
m_{1}^{N}=1 \\
m_{2}^{N}=N-1 \\
m_{3}^{N}=\sum_{k=2}^{N-2} k \quad(N-2>2) \\
m_{3+j}^{N}=\sum_{K=3+2 j}^{N-1} m_{3+j-1}^{K} \quad j>0, \quad N-1 \geq 3+2 j
\end{gathered}
$$

E.g. for $N=6,7,8,9$ we have, omitting the upper label

$$
\begin{gathered}
m_{3}=9 \quad m_{4}=5 \\
m_{3}=14 \quad m_{4}=14 \\
m_{3}=20 \quad m_{4}=28 \quad m_{5}=14 \\
m_{3}=27 \quad m_{4}=48 \quad m_{5}=42
\end{gathered}
$$

(Recall that $J^{(i)}=\mathbf{i} / \mathbf{2}$ ) 


\section{References}

Arndt P.F., Burge C.B. and Hwa T., 2002, DNA Sequence Evolution with Neighbor-Dependent Mutation, RECOMB2002, Proc. 6th Int.Conf. on Computational Biology (2002), p.32 (physics/0112029). Blake R.D., Hess S.T. and Nicholson-Tuell J., 1992, The influence of Nearest Neighbors on the Rate and Pattern of Spontaneous Mutations, J.Mol.Evol. 34, 189.

Blake R.D., Hess S.T. and Blake R.D., 1994, Wide Variations in Neighbor-dependent Substitution Rates , J.Mol.Biol. 236, 1022.

Baake E., Baake M. and Wagner H., 1997, Ising quantum chain is equivalent to a model of biologicalevolution, Phys.Rev.Lett. 78 , 559; Erratum Phys.Rev.Lett. 79 , 1782.

Baake E., Baake M. and Wagner H., 1998, Quantum mechanics versus classical probability in biological evolution, Phys.Rev. E 57, 1191.

Baake E. and Wagner H., 2001, Mutation-selection models solved exactly with methods of statistical mechanics, Genet.Res.Camb. 78, 93.

Blake R.D., Hess S.T. and Nicholson-Tuell J., 1992, The Influence of Nearest Neighbors on the Rate and Pattern of Spontaneous Point Mutation, J.Mol.Evol. 34, 189.

Eigen M., 1971, Selforganisation of matter and the evolution of biological macromolecules, Naturwissenschaften 58, 465.

Eigen M., McCaskill J. and Schuster P., 1989, The molecular quasi-species, J.Chem.Phys. 75, 149. Encyclopedic Dictionary of Mathematics, 1960, 2nd edition, The MIT Press, Cambridge, (MA).

Frappat L., Sciarrino A. and Sorba P., 1998, A crystal basis model of the genetic code Phys. Lett. A 250214.

Frappat L., Sciarrino A. and Sorba P., 2001, Crystalizing the genetic code J.Biol. Phys. 271.

Frappat L., Minichini C., Sciarrino A. and Sorba P., 2003, Universality and Shannon Entropy for Codon Usage Phys.Rev. E 68, 061910.

Goldstein RF. and Benight A. S., 1992, How Many Numbers Are Required to Specify SequenceDependent Properties of Polynucleotides?, Biopolymers 32, 1679.

Hermisson J., Wagner H. and Baake M., 2001, Four-State Quantum Chain as a Model of Sequence Evolution, J.Stat.Phys. 102, 315.

Hermisson J., Redner O., Wagner H. and Baake E., 2002, textitMutation-selection balance: Ancestry, load and maximum priciple, Theoretical Population Biology 62, 9.

Hess S.T., Blake J.D. and Blake R.D., 1994, Wide Variations in Neighbor-dependent Substitution Rates, J.Mol.Evol. 236, 1022.

Hofbauer J. and Sigmund K., 1988, The Theory of Evolution and Dynamical Systems. Cambridge University Press, Cambridge.

Kashiwara M., 1990, Crystallizing the q-analogue of universal enveloping algebras, Commun. Math. Phys. 133249. 
Kirillov A.A., 1991, Representation theory and Noncommutative Harmonic Analysisis, Encyclopedia of Mathematical Sciences, Vol. 22, pag. 70, Springer Verla, Berlin.

Leuthäusser I., 1986, An exact correspondence between Eigen's evolution model and a two-dimensional Ising system, J.Chem.Phys. 84, 1884.

Leuthäusser I., 1987, Statistical mechanics of Eigen's evolution model, J.Stat.Phys. 48, 343.

Li Wen-Hsiung, 1997, Molecular Evolution, Sinauer Associates Incorporated, Sunderland.

Martindale C. and Konopka A. K., 1996, Oligonucleotide Frequencies in DNA Follow a Yule Distributiojn, Computers Chem. 20, 35.

Minichini C. and Sciarrino A., 2004a, Quantum Spin Model fitting the Yule distribution of oligonucleotides, quant-ph/0409071

Minichini C. and Sciarrino A., 2004b, Mutation Model fitting the Yule distribution of oligonucleotides, q-bio.BM/0412006

Saakian S. and Hu Chin-Kun, 2004, Eigen Model as a Quantum Spin Chain: Exact Dynamics, Phys.Rev. E 69, 021913 (cond-mat/0402212).

SantaLucia, J., 1998, A unified view of polymer, dumbbell, and oligonucleotide DNA nearest-neighbor thermodynamics, Proc.Natl.Acad.Sci USA 95, 1460.

Wagner H., Baake E. and Gerisch T., 1998, Ising quantum chain and sequence evolution, J.Stat.Phys. $92,1017$.

Whelan S. and Goldman N., 2004, Estimating the Frequency of Events That Cause Multiple-Nucleotide Changes, Genetics 167, 2027.

Xia Tiambing et al, 1998, Thermodynamic Parameters for an Expanded Nearest-Neighbor Model for Formation of RNA Duplexes with Watson-Crick Base Pairs, Biochemistry 37, 14719.

Yule G.U., 1924, A mathematical theory of evolution, based on the conclusions of Dr.J.C. Willis, F.R.S., Phil.Trans. B 213, 21.

Zipf, G.K., 1949 Human behaviour and the Principle of Least Effort, Addison-Wesley Press, Cambridge, $M A$. 


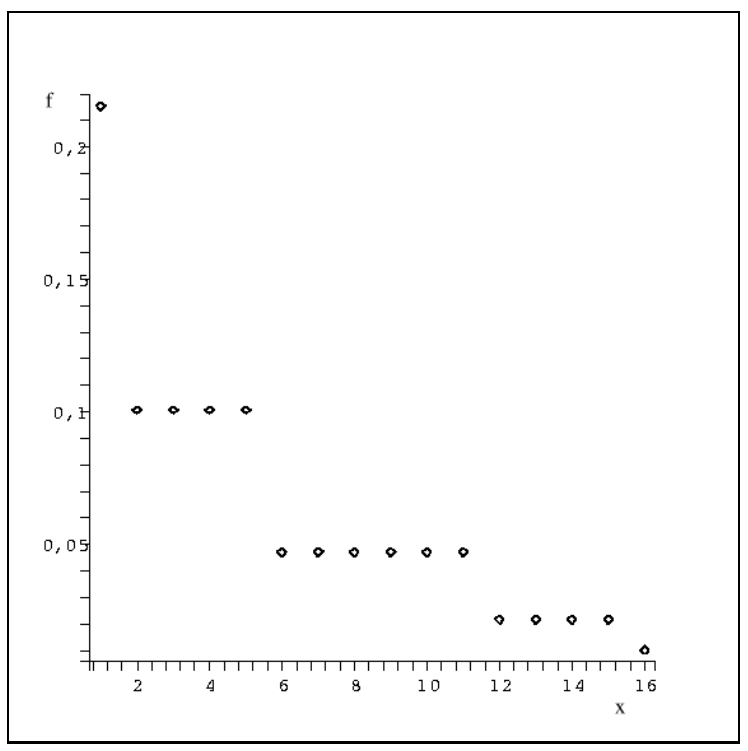

Figure 1: Rank ordered distribution of equilibrium population $(\mathrm{N}=4)$ obtained for a Hamming transition matrix, with $\alpha=0.60$.

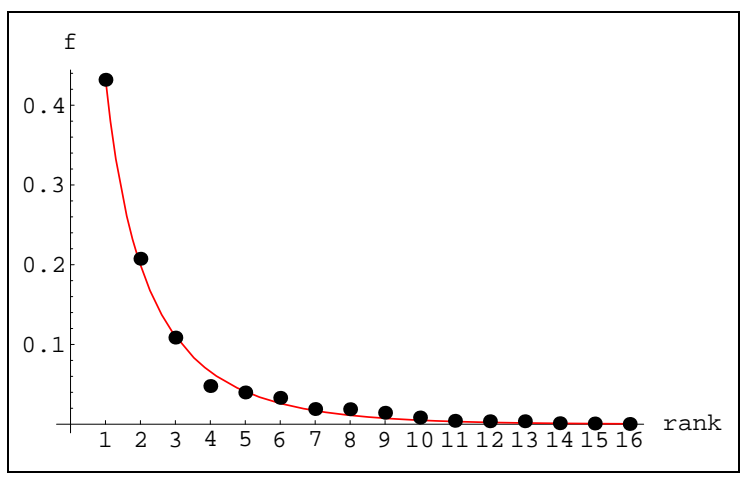

Figure 2: Rank ordered distribution of equilibrium population $(\mathrm{N}=4)$ for a transition matrix $M$ with $\epsilon=0.25, \gamma=\delta=\eta=0.50$. The distribution was fitted by a Yule function (continuous line) $f=a R^{k} b^{R}$ ( $R$ is the rank). The parameters were estimated as $a=0.37, b=1.02, k=-1.28$. 


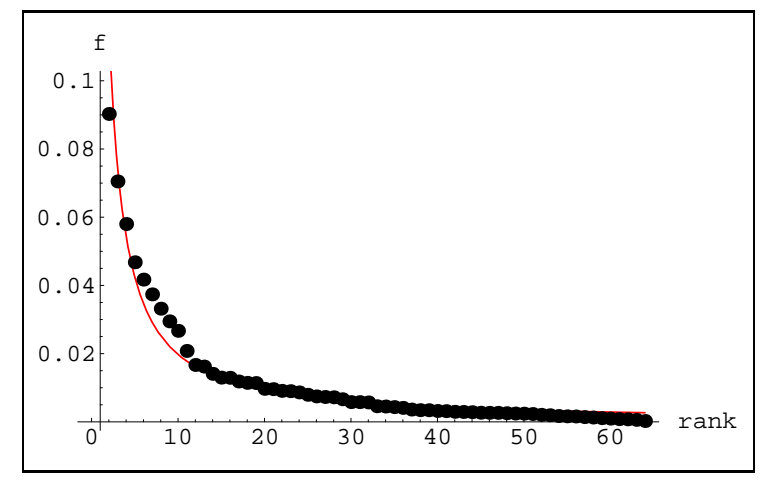

Figure 3: Rank ordered distribution of equilibrium population $(\mathrm{N}=6)$ for a transition matrix $M$, with $\epsilon=0.25, \gamma=\delta=\eta=0.50$. The distribution was fitted by a Yule function (continuous line) $f=a R^{k} b^{R}$ ( $R$ is the rank). The parameters were estimated as $a=0.26, b=1.00 k=-1.11$.

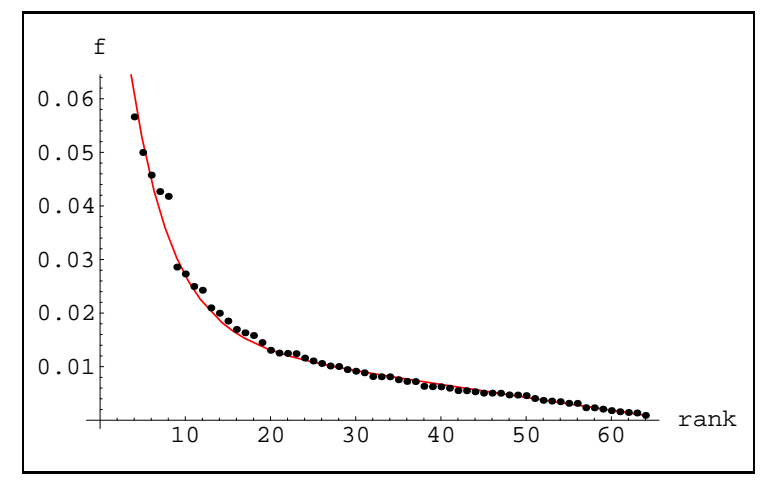

Figure 4: Rank ordered distribution of equilibrium population of codons, for the set of parameters $\mu_{1, H}=1.2, \mu_{2, H}=\mu_{3, H}=3.6, \mu_{1, V}=0.4, \mu_{2, V}=\mu_{3, V}=1.2$. The distribution was fitted by the function $f(n)=\alpha \exp (-\eta n)-\beta n+\gamma$ (continuous line), where $n$ is the rank of the codons. The parameters were estimated as $\alpha=0.084, \beta=2.77 \cdot 10^{-4}, \gamma=0.018, \eta=0.219$.

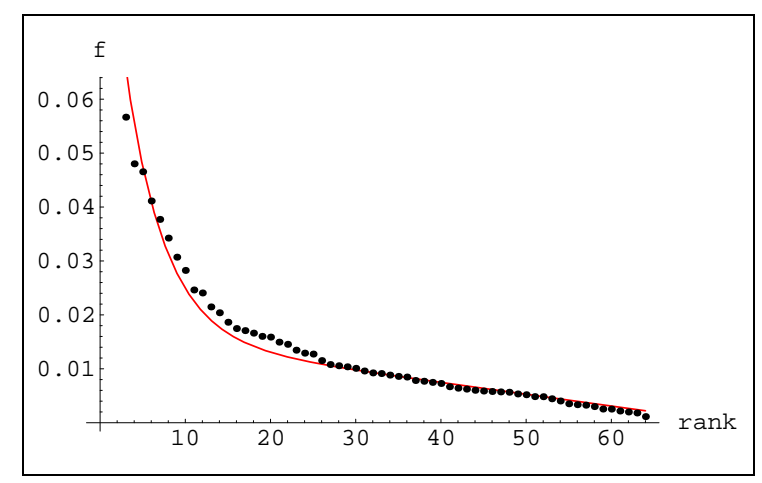

Figure 5: Rank ordered distribution of equilibrium population of codons, for the set of parameters $\mu_{1, H}=1, \mu_{2, H}=\mu_{3, H}=3, \mu_{1, V}=0.5, \mu_{2, V}=\mu_{3, V}=1.5$. The distribution was fitted by the function $f(n)=\alpha \exp (-\eta n)-\beta n+\gamma$ (continuous line), where $n$ is the rank of the codons. The parameters were estimated as $\alpha=0.076, \beta=3.46 \cdot 10^{-4}, \gamma=0.022, \eta=0.285$. 


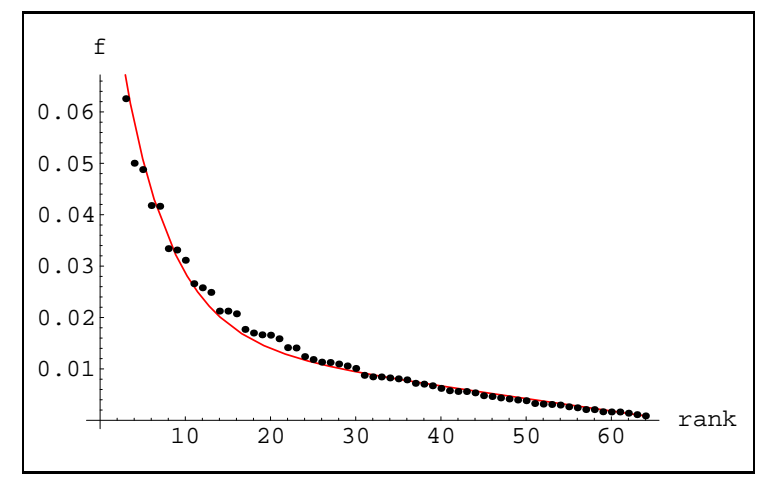

Figure 6: Rank ordered distribution of equilibrium population of codons, for the set of parameters $\mu_{1, H}=\mu_{2, H}=3, \mu_{3, H}=1, \mu_{1, V}=\mu_{2, V}=1, \mu_{3, V}=0.3$. The distribution was fitted by the function $f(n)=\alpha \exp (-\eta n)-\beta n+\gamma$ (continuous line), where $n$ is the rank of the codons. The parameters were estimated as $\alpha=0.071, \beta=3.36 \cdot 10^{-4}, \gamma=0.020, \eta=0.211$.

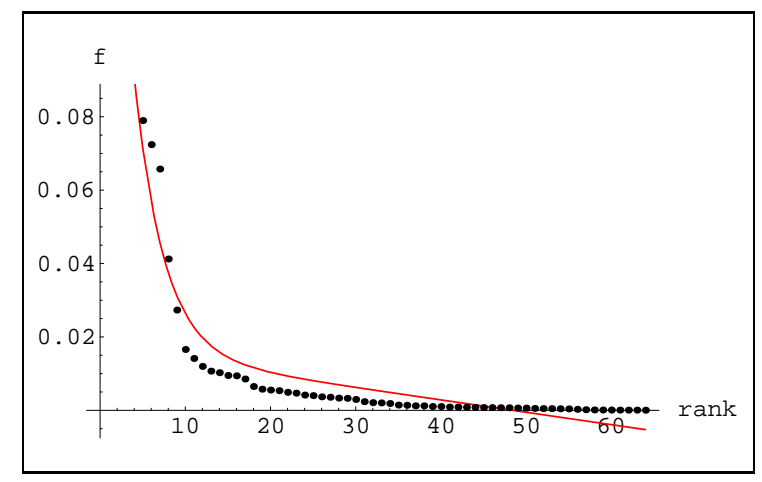

Figure 7: Rank ordered distribution of equilibrium population of codons, for the set of parameters $\mu_{1, H}=1, \mu_{2, H}=\mu_{3, H}=3, \mu_{1, V}=0.1, \mu_{2, V}=\mu_{3, V}=0.3$. The distribution was fitted by the function $f(n)=\alpha \exp ^{-\eta n}-\beta n+\gamma$ (continuous line), where $n$ is the rank of the codons. The parameters were estimated as $\alpha=0.187, \beta=8.75 \cdot 10^{-5}, \gamma=0.005, \eta=0.246$.

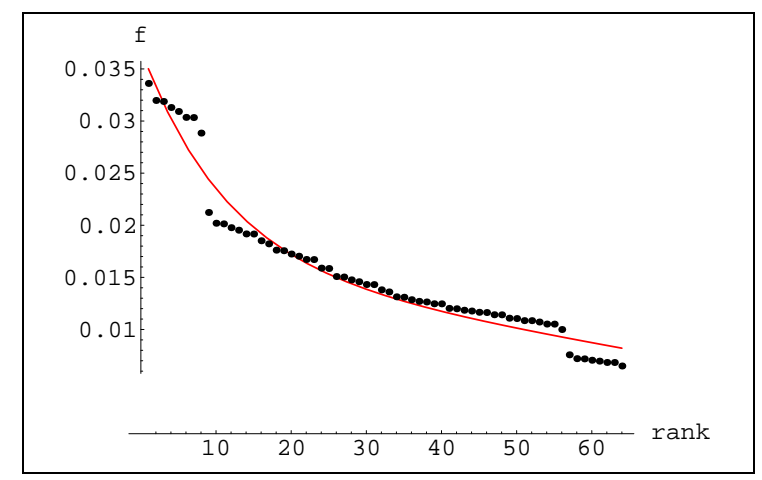

Figure 8: Rank ordered distribution of equilibrium population of codons, for the set of parameters $\mu_{1, H}=1.1, \mu_{2, H}=\mu_{3, H}=3.3, \mu_{1, V}=11, \mu_{2, V}=\mu_{3, V}=33$. The distribution was fitted by the function $f(n)=\alpha \exp (-\eta n)-\beta n+\gamma$ (continuous line), where $n$ is the rank of the codons. The parameters were estimated as $\alpha=0.016, \beta=1.87 \cdot 10^{-4}, \gamma=0.019, \eta=0.122$. 


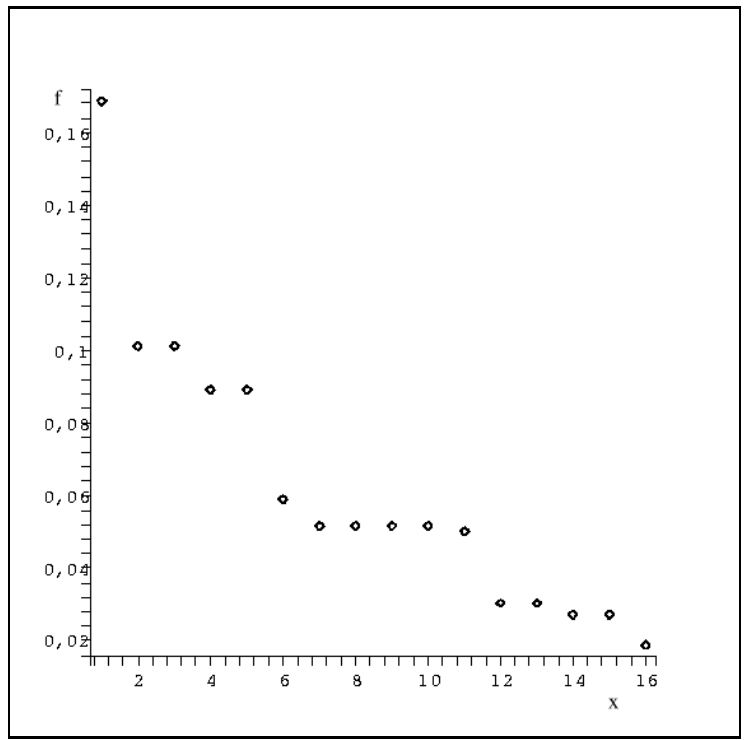

Figure 9: Rank ordered distribution of equilibrium population $(\mathrm{N}=4)$ obtained for a transition matrix allowing the same number of mutations as $M$, between sequences at different Hamming distances 